\title{
Traveling wave solution of the Reggeon Field Theory
}

\section{Robi Peschanski*}

Institut de Physique Théorique and URA 2306, Unité de Recherche associée au CNRS,

CEA-Saclay, F-91191 Gif/Yvette Cedex, France

E-mail: robi.peschanskilcea.fr

We identify the nonlinear evolution equation in impact-parameter space for the "Supercritical Pomeron" in Reggeon Field Theory as a 2-dimensional stochastic Fisher and KolmogorovPetrovski-Piscounov equation. It exactly preserves unitarity and leads in its radial form to an high energy traveling wave solution corresponding to an "universal" behaviour of the impact-parameter front profile of the elastic amplitude; Its rapidity dependence and form depend only on one parameter, the noise strength, independently of the initial conditions and of the non-linear terms restoring unitarity. Theoretical predictions are presented for the three typical different regimes corresponding to zero, weak and strong noise, respectively. They have phenomenological implications for total and differential hadronic cross-sections at colliders.

European Physical Society Europhysics Conference on High Energy Physics, EPS-HEP 2009,

July 16 - 222009

Krakow, Poland

${ }^{*}$ Speaker. 


\section{From Reggeon Field Theory (1967-1975) to the stochastic sFKPP Equation}

Reggeon Field Theory (RFT) has been motivated, long ago, by an effective field-theoretical treatment [1] of the Strong Interaction physics at high-energy, commonly called nowadays "soft interactions", since they involved only rather small transfer of momentum. As such, they belong to the strongly-coupled regime of QCD and thus not reducible to perturbative calculations. The field-theoretical action is [1]

$$
S[\bar{\varphi}, \varphi]=\frac{1}{\alpha^{\prime}} \int d^{2} b d Y\left\{\bar{\varphi}\left[\partial_{Y}-\alpha^{\prime} \nabla^{2}\right] \varphi-\mu \bar{\varphi} \varphi+g\left(\bar{\varphi} \varphi^{2}-\kappa \bar{\varphi}^{2} \varphi\right)\right\},
$$

where $\varphi(Y, \vec{b})$ is the Reggeon field. It is considered as a non-relativistic quantum field moving in a (1+2)-dimensional space spanned by $Y$, the total rapidity considered as a time variable and $\vec{b}$ the impact-parameter vector in the transverse plane of the interaction. $\alpha^{\prime}$ is the Regge slope, $\mu+1$ is the Reggeon intercept. The coupling constants $g$ and $g \kappa$ characterize the Reggeon self interactions, such that $g$ is the coupling strength for the $2 \rightarrow 1$ merging while $g \kappa$ defines the $1 \rightarrow 2$ splitting vertex. Note that $\kappa=1$ recovers the original symmetric RFT model [1]. The field-theoretical formalism relies on the introduction of an auxiliary reggeon field $\bar{\varphi}$ which plays the role of a source term. It is important to realize that the theory is nonhermitian $(\bar{\varphi} \neq \varphi \dagger)$ which is at the root of a deep relation with out-of-equilibrium statistical physics of reaction-diffusion type, as we shall see.

We are interested in the so-called "Supercritical" Reggeon problem where the Born term (corresponding to retaining only the quadratic part of (1.1)) reads

$$
\varphi(Y, \vec{b})=\exp \mu Y-\frac{b^{2}}{4 \alpha^{\prime} Y} ; \quad \mu>0,
$$

which has been shown to be phenomenologically preferred but for which no theoretical solution has yet been found for the corresponding RFT. Our goal is to show that, using an intimate connection with statistical physics one is able [2] to find the solution of the RFT giverned by the action (1.1).

Using the Stratonovitch transformation to linearize the action as a function of the auxiliary field $\bar{\varphi}$ and performing the functional integral $\int \mathscr{D} \bar{\varphi} \mathscr{D} \varphi \exp \left(\frac{i}{\alpha^{\prime}} S[\bar{\varphi}, \varphi]\right)$ one gets [2] a nonlinear Langevin equation for the Reggeon solution $\varphi(Y, \vec{b})$. After reparametrisation, the equation boils down to

$$
\partial_{t} \varphi(t, \vec{r})=\nabla_{r}^{2} \varphi+\varphi-\varphi^{2}+\varepsilon \sqrt{\varphi(1-\varphi)} v(t, \vec{r})
$$

where $v(t, \vec{r})$ is the two-dimensional white noise. The dictionnary between the RFT and sFKPP is

$$
\text { Time }: t=\mu\left(Y-Y_{0}\right) ; \quad \text { Space }: \vec{r}=\frac{\mu}{\alpha^{\prime}} \vec{b} ; \quad \text { Noise }: \varepsilon=\sqrt{2 \mu \kappa} .
$$

Eq.(1.3) is nothing else than the extension in 2 dimensions of the known stochastic Fisher and Kolmogorov, Petrovsky, Piscounov equation (sFKPP).

In this way, the RFT is mapped onto an equation describing non-equilibrium processes in a 2dimensional space with diffusion, creation, merging and splitting as a function of time. In Eq.(1.3), all coupling strengths characterizing these processes can be absorbed in the redefinitions (1.4), except for the parameter $\varepsilon=\sqrt{2 \mu \kappa}$ which thus appears as the only free parameter. 


\section{Traveling waves and the RFT "Phase Diagram"}

In the problem of deriving solutions for the azimuthally symmetric Reggeon amplitude, the 2-dimensional problem can be further reduced to a radial equation, with $r \equiv|\vec{r}|$

$$
\partial_{t} \varphi(t, r)=\partial_{r r} \varphi+\frac{1}{r} \partial_{r} \varphi+\varphi-\varphi^{2}+\varepsilon \sqrt{\frac{\varphi(1-\varphi)}{2 \pi r}} v(t, r) .
$$

We are thus led to consider asymptotic solutions of the 1-d radial sFKPP equation.

The main property of the $1 \mathrm{~d}$ sFKPP equations [3] is the existence of asymptotic traveling wave solutions $\varphi(t, r) \rightarrow \varphi(r-r(t))$ characterized by a wave front $r(t) \sim v t$ where the solution jumps from the value 1 to 0 and moving with the speed $v$. Apart from the $\frac{1}{r} \partial_{r} \varphi$ term which is subdominant at large $r$, the main change w.r.t. the standard $1 \mathrm{~d}$ sFKPP equation is in the noise term which is now characterized by an effective coupling strength

$$
\zeta \sim \frac{\varepsilon}{\sqrt{2 \pi r(t)}}=\sqrt{\frac{\kappa \sqrt{\alpha^{\prime} \mu}}{2 \pi b_{s}(Y)}},
$$

where we have approximated the $r$-dependence of the noise by its value at the wave front (where $\varphi(1-\varphi)$ is not small) and $b_{s}(Y) \equiv \alpha^{\prime} / \mu r(t)$ is the effective impact-parameter radius. Note that the noise (2.2) is reduced by a factor of order $\sqrt{(v t)}$ compared to the 2d-sFKPP equation due to the azimuthal symmetry, since it has to be restricted to azimuthally symmetric fluctuations.

The typical structure of the solutions for the wave speed is displayed (for the $1 \mathrm{~d}$ sFKPP equation [4]) in Fig.1. The vertical axis is the speed of the wave front $v / v_{0}$ normalized by the no-noise speed $v_{0} \equiv 2$. The horizontal axis is the normalized noise strength, represented by $\zeta$ in the approximation (2.2). One is led to distinguish mainly three different regimes of traveling wave solutions which may play the role of a RFT phase diagram depending on the noise strength.

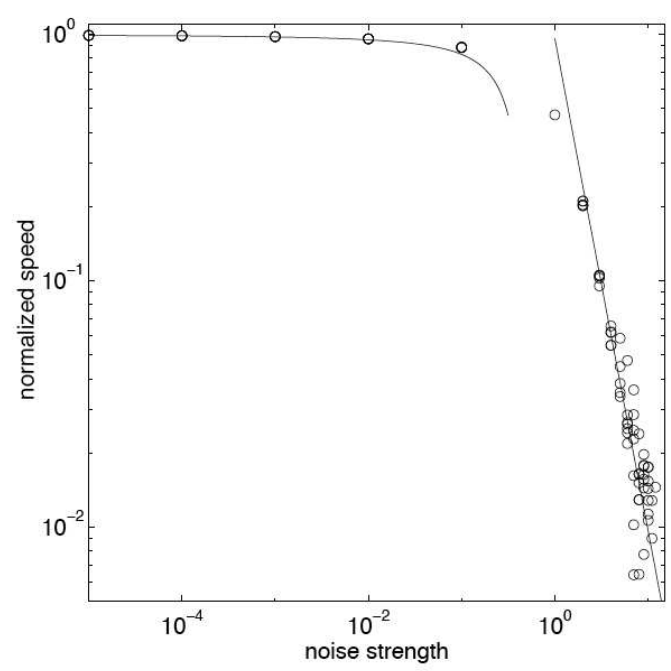

Figure 1: Normalized wave speed as a function of the normalized noise strength (from Ref. [4]). White dots: numerics; Lines: analytic results. 
Let us restore the appropriate high-energy observables, namely $b_{s}(Y)$, the impact-parameter radius, $\sigma_{t o t} \propto b_{s}^{2}(Y)$, the total cross-section, and $T(Y, b)$, the impact-parameter dependence of the imaginary elastic amplitude and discuss the three different regimes appearing in Fig.1.

The first "phase" is the no-noise regime where $\zeta \sim \kappa \approx 0$, i.e. when splitting $\ll$ merging. One gets,

$$
\sigma_{t o t} \propto b_{s}^{2}(Y)=\left\{b_{0}+2 \sqrt{\alpha^{\prime} \mu}\left[Y-Y_{0}-\frac{1}{\mu} \log \frac{Y}{Y_{0}}+\cdots\right]\right\}^{2} ; \quad T(Y, b) \sim T\left(b-b_{s}(Y)\right) .
$$

The second phase is the so-called "weak-noise" phase where $\kappa \approx \mathscr{O}(1)$, and splitting $\sim$ merging, corresponding to the original RFT action (1.1) and leading to

$$
\sigma_{t o t}=\left\{b_{0}+2 \sqrt{\alpha^{\prime} \mu}\left(Y-Y_{0}\right)\left[1-\frac{\pi^{2}}{2 \log ^{2}\left(2 \zeta^{-2}\right)}\right]\right\}^{2} ; T(Y, b) \sim T\left(\frac{b-b_{s}(Y)}{D \sqrt{\alpha^{\prime}\left(Y-Y_{0}\right)}}\right) .
$$

The third phase, corresponding to "strong noise" with $\kappa \approx \mathscr{O}(100)$, and splitting $\gg$ merging gives

$$
\sigma_{t o t}=b_{s}^{2}\left(Y_{0}\right) \exp \left[\frac{8 \pi}{\kappa}\left(Y-Y_{0}\right)\right] ; \quad T(Y, b) \sim \operatorname{erfc}\left(\frac{b-b_{s}(Y)}{D \sqrt{\alpha^{\prime}\left(Y-Y_{0}\right)}}\right) .
$$

It is interesting to note that, due to the energy dependence $\zeta \propto 1 / \sqrt{Y}$, see (2.2), there is a natural evolution from strong to weak and ultimately no-noise "phases" as a function of energy. Hence, for instance, the apparently exponential growth of the cross-section (2.5) at strong noise, which would violate the Froissart bound, is tamed by the noise strength evolution transfering the energy behaviour into the $Y^{2}$ behaviour of (2.4) and finally ending into (2.3).

Let us summarize the main consequences of our study [2]:

- The supercritical Reggeon Field Theory can be exactly mapped onto the $2 \mathrm{~d}$ stochastic Fisher and Kolmogorov-Petrovski-Piscounov equation.

- The solution for the elastic amplitude at high-energy is given by universal traveling wave solutions of the radial sFKPP equation.

- A "Phase Diagram" depending only on the ratio between the splitting over merging coupling of the Reggeons plays the role of a dynamical "order parameter", characterizing the solution.

It is to be remarked that the asymptotic behaviour does not depend on the rather strong renormalization of the "supercritical" Born term (1.2). Thinking in terms of Regge singularities, such universality, observed experimentally in hadron-hadron reactions and usually associated to the Regge pole structure of the Born term (1.2), is here restored through the universality of traveling wave solutions while the Born term is completely masked by Reggeon cuts through unitarity restoration. The final word is now to be given to phenomenology.

\section{References}

[1] H. D. I. Abarbanel, J. B. Bronzan, R. L. Sugar and A. R. White, Phys. Rept. 21, 119 (1975).

[2] R. Peschanski, Phys. Rev. D 79, 105014 (2009).

[3] R. A. Fisher, Ann. Eugenics 7, 355 (1937); A. Kolmogorov, I. Petrovsky, and N. Piscounov, Moscou Univ. Bull. Math. A1, 1 (1937).

[4] C. R. Doering, C, Mueller and P. Smereka, Phys. A 325, 243 (2003). 